\title{
An Efficient Bit Loading for OFDM with Diversity Scheme over Mobile Channel
}

\author{
Tae Jin Hwang, Sang Soon Park, and Ho Seon Hwang \\ 664-14, Duckjin-Dong 1Ga, Jeonju 561-756, Korea \\ Department of Electronic Engineering, Chonbuk National University \\ tjhwang@chonbuk.ac.kr
}

\begin{abstract}
This paper discusses an adaptive modulation technique combined with space-frequency block coded OFDM(SFBC OFDM) over frequency selective channels and evaluates the performance in terms of the outdated channel state information(CSI) in mobile environments. This paper employs the Alamouti's diversity scheme in multiple input multiple output OFDM (MIMO OFDM) and an adaptive modulation with enhanced performance. Adaptive modulation scheme shows very attractive performance when the CSI is perfect. The CSI for bit loading in MIMO OFDM can be obtained from the singular value decomposition(SVD) of MIMO channel. But, SFBC OFDM system based on Alamouti's diversity scheme does not require the SVD process. Through various simulations, the performance of SFBC OFDM employing adaptive modulation is compared with that of fixed modulation. Also, in adaptive modulation scheme, the effects of the outdated CSI under mobile environments are shown
\end{abstract}

\section{Introduction}

Recently, a considerable number of studies have been conducted on reliable high data rate services in broadband wireless communications. In frequency selective channel, some of mechanisms are required in order to combat the effects of intersymbol interference(ISI). Orthogonal frequency division multiplexing(OFDM) technique transforms a frequency selective channel into parallel correlated flat channels by increasing symbol duration. However, the performance of OFDM system could be degraded due to deep faded subchannels. For a better performance, the OFDM transmitter adapts the subchannel bit and power allocation to the amplitude response of the frequency selective channel[1].

On the other hand, the most popular technique has been the exploitation of diversity in order to overcome multipath fading in wireless environments. Alamouti discovered a remarkable space time block coded scheme using two transmit antennas in narrowband wireless communication [2]. The novel diversity scheme has been proved effectively in combating fading and has motivated various transmit diversity techniques. However, this is faced with a very complex equalization problem to overcome frequency-selective fading environments. Several studies have been made on transmit diversity technique in context of OFDM, such as space-time block coded OFDM (STBC OFDM) or space-frequency block coded OFDM(SFBC OFDM), and et al [3] [5]. 
In order to improve the performance of OFDM system in frequency selective and multipath fading environments, this paper presents an adaptive bit allocation combined with SFBC OFDM. The perfect CSI ensures a desired efficiency/ performance of adaptive modulation scheme. In MIMO OFDM system, by making use of SVD the MIMO channel on each subcarrier is decomposed into parallel noninterfering single input single output(SISO) channels. But, a SFBC OFDM system with Alamouti's diversity scheme does not require the SVD for the CSI. Assuming the availability of the perfect CSI at the transmitter, the performance gains of adaptive modulation have been demonstrated. This paper examines the impact on performance of an adaptive OFDM system, which combined with SFBC scheme, due to the outdated CSI in mobile fading channel. The organization of this paper is as follows. In section II, we will describe a system model and introduce SFBC OFDM in brief. In section III, we discuss the considered CSI for adaptive modulation in SFBC OFDM system and explain bit allocation method. In section IV, the performance of adaptive SFBC OFDM is evaluated under a variety of environment. Finally, a conclusion is made in Section V.

\section{System Model and SFBC-OFDM}

\subsection{System Description}

Fig.1 illustrates a block diagram of SFBC OFDM with adaptive modulation. To begin with, we assume that both the transmitter and the receiver perfectly know the channel state information. But, in mobile situation, the CSI will be outdated at the transmission time. In this paper, we consider the Alamouti's transmit diversity scheme applied to MIMO OFDM system with $N$ tones, two transmit antennas and two receive antennas. At the transmitter, adaptive modulation based on the CSI is firstly performed. Two blocks of data through adaptive modulator are serial to parallel $(\mathrm{S} / \mathrm{P})$ converted. Two symbol vectors, $\mathbf{X}_{1}$ and $\mathbf{X}_{2}$, are coded by ST encoder. During the $n$ th symbol period, the symbol vector $\mathbf{X}_{1}$ will be transmitted from antenna one and $\mathbf{X}_{2}$ will be transmitted from antenna two. During next symbol period, $-\mathbf{X}_{2}^{*}$ will be

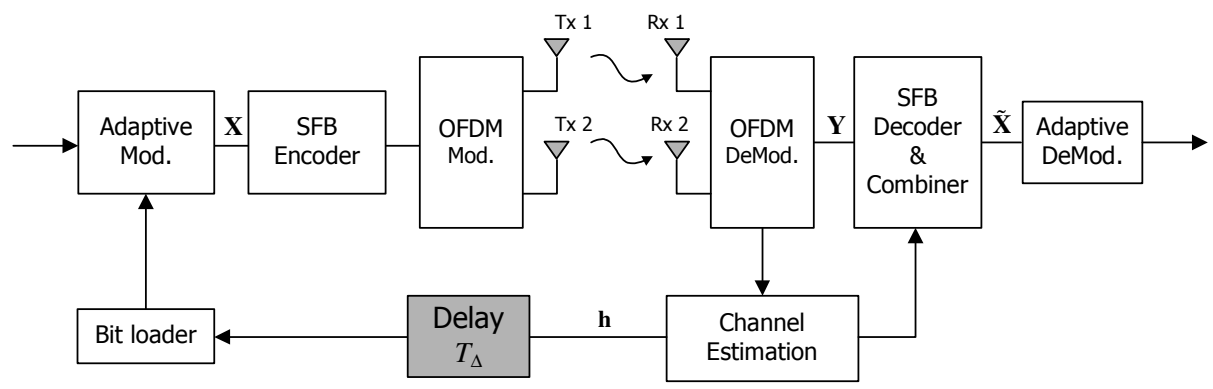

Fig. 1. Block diagram of an adaptive SFBC OFDM 
transmitted from antenna one, and $\mathbf{X}_{1}^{*}$ will be transmitted from antenna two where $*$ is the complex conjugate operation. The respective vectors are synthesized by the inverse fast Fourier transform (IFFT). To avoid ISI, a cyclic prefix (CP) is inserted at the beginning of each OFDM frame as a guard interval, the length of which is at least as long as the maximum delay of two channels. Two signals are transmitted at a particular transmitter antenna during two time slots. The transmitted signal is faded by time varying channel and added noise. At the receiver, the transmitted symbols are sequentially restored through the maximal ratio combiner(MRC), maximum likelihood(ML) detector and adaptive demodulator.

\subsection{Space Frequency Block Coded OFDM Scheme}

This paper considers a MIMO OFDM system employing the Alamouti's diversity scheme. To begin with, let $\mathbf{X}(n)$ be the $n$-th transmit symbol as follows

$$
\mathbf{X}(n)=[X(n, 0) X(n, 1) \cdots X(n, N-1)]^{T}
$$

The data symbol vector $\mathbf{X}(n)$ is coded into two vectors $\mathbf{X}_{1}(n)$ and $\mathbf{X}_{2}(n)$ by the space-frequency encoder block as

$$
\begin{aligned}
& \mathbf{X}_{1}(n)=\left[\begin{array}{lllll}
X(n, 0) & -X^{*}(n, 1) & \cdots & X(n, N-2) & -X^{*}(n, N-1)
\end{array}\right]^{T} \\
& \mathbf{X}_{2}(n)=\left[\begin{array}{lllll}
X(n, 1) & X^{*}(n, 0) & \cdots & X(n, N-1) & X^{*}(n, N-2)
\end{array}\right]^{T} .
\end{aligned}
$$

At the $n$th block, $\mathbf{X}_{1}(n)$ is transmitted from the first base station Tx1 while $\mathbf{X}_{2}(n)$ is transmitted simultaneously from the second base station Tx2. The operations of the space frequency encoder/decoder can be described in terms of even and odd poly-phase component vectors. Let $\mathbf{X}_{e}(n)$ and $\mathbf{X}_{o}(n)$ be two vectors denoting the even and odd component vectors of $\mathbf{X}(n)$. Similarly, $\mathbf{X}_{1, e}(n)$ and $\mathbf{X}_{1, o}(n)$ denote the even and odd component vectors of $\mathbf{X}_{1}(n)$, i.e.,

$$
\begin{aligned}
& \mathbf{X}_{1, e}(n)=\left[\begin{array}{lll}
X(n, 0) & \cdots & X(n, N-2)
\end{array}\right]^{T} \\
& \mathbf{X}_{1, o}(n)=\left[\begin{array}{lll}
-X^{*}(n, 1) & \cdots & -X^{*}(n, N-1)
\end{array}\right]^{T} .
\end{aligned}
$$

Also, $\mathbf{X}_{2, e}(n)$ and $\mathbf{X}_{2, o}(n)$ denote the even and odd component vectors of $\mathbf{X}_{2}(n)$,i.e.

$$
\begin{aligned}
& \mathbf{X}_{2, e}(n)=\left[\begin{array}{lll}
X(n, 1) & \cdots & X(n, N-1)
\end{array}\right]^{T} \\
& \mathbf{X}_{2, o}(n)=\left[\begin{array}{lll}
X^{*}(n, 0) & \cdots & X^{*}(n, N-2)
\end{array}\right]^{T} .
\end{aligned}
$$

Therefore, $\mathbf{X}_{e}(n)$ and $\mathbf{X}_{o}(n)$ can be expressed in terms of upper vectors as

$$
\begin{aligned}
& \mathbf{X}_{e}(n)=\mathbf{X}_{1, e}(n)=\mathbf{X}_{2, o}^{*}(n) \\
& \mathbf{X}_{o}(n)=\mathbf{X}_{2, e}(n)=-\mathbf{X}_{1, o}^{*}(n) .
\end{aligned}
$$


Let $\mathbf{H}_{i j}(n)$ be the following diagonal matrix whose diagonal elements are the frequency responses of the channel impulse responses $h_{i j}$ between the $i$-th transmit antenna and the $j$-th receive antenna during the $n$-th time slot

$$
\mathbf{H}_{i j}(n)=\operatorname{diag}\left[H_{i j}(n, 0) \cdots H_{i j}(n, N-1)\right], \quad i=1,2, j=1,2
$$

Let $\mathbf{Y}_{j}(n)$ be the $n$-th received OFDM symbol from the $j$-th receive antenna as follows

$$
\mathbf{Y}_{j}(n)=\mathbf{H}_{1 j}(n) \mathbf{X}_{1}(n)+\mathbf{H}_{2 j}(n) \mathbf{X}_{2}(n)+\mathbf{W}_{j}(n), \quad j=1,2 .
$$

\section{Adaptive Modulation in SFBC OFDM}

\subsection{Parallel Decomposition of the MIMO Channel Using SVD}

Fig. 2 shows the equivalent model of MIMO OFDM. At the receiver, the $k$-th demodulated subcarrier vector in the $n$-th time slots is given by

$$
\mathbf{R}(n, k)=\mathbf{h}(n, k) \mathbf{S}(n, k)+\mathbf{W}(n, k)
$$

where $\mathbf{R}(n, k)=\left[R_{1}(n, k) R_{2}(n, k)\right]^{T}$ and the additive white Gaussian noise is $\mathbf{W}(n, k)=\left[W_{1}(n, k) W_{2}(n, k)\right]^{T}$. The channel matrix $\mathbf{h}(n, k)$ is as follows

$$
\mathbf{h}(n, k)=\left[\begin{array}{ll}
H_{11}(n, k) & H_{21}(n, k) \\
H_{12}(n, k) & H_{22}(n, k)
\end{array}\right]
$$

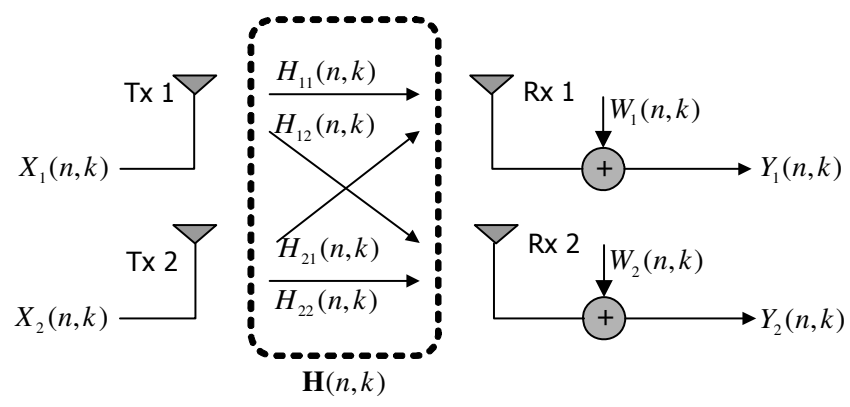

Fig. 2. An equivalent model of MIMO OFDM

We assume that both the transmitter and the receiver know $\mathbf{h}(n, k)$ at each instant. Let the instantaneous channel matrix have singular value decomposition

$$
\mathbf{h}(n, k)=\mathbf{U}(n, k) \boldsymbol{\Lambda}(n, k) \mathbf{V}^{H}(n, k)
$$


where $\mathbf{U}(n, k)$ and $\mathbf{V}(n, k)$ are unitary matrices, the matrix $\mathbf{V}^{H}(n, k)$ means the hermitian matrix of $\mathbf{V}(n, k)$ and $\boldsymbol{\Lambda}(n, k)$ is the diagonal matrix of singular values of $\mathbf{h}(n, k)$, i.e., $\boldsymbol{\Lambda}(n, k)=\operatorname{diag}\left[\lambda_{1}(n) \lambda_{2}(n)\right]$ with $\lambda_{1}(n) \geq \lambda_{2}(n) \geq 0$. By transmit $\operatorname{precoding}\left(\overline{\mathbf{S}}=\mathbf{V}^{H} \mathbf{S}\right)$ and receiver shaping $\left(\overline{\mathbf{R}}=\mathbf{U}^{H} \mathbf{R}\right)$, the MIMO channel is transformed into parallel single input single output channels

$$
\begin{aligned}
\overline{\mathbf{R}}(n, k) & =\mathbf{U}^{H}(n, k) \mathbf{R}(n, k) \\
& =\mathbf{U}^{H}(n, k) \mathbf{h}(n, k) \overline{\mathbf{S}}(n, k)+\overline{\mathbf{W}}(n, k) \\
& =\boldsymbol{\Lambda}(n, k) \mathbf{S}(n, k)+\overline{\mathbf{W}}(n, k)
\end{aligned}
$$

where $\overline{\mathbf{W}}=\mathbf{U}^{H} \mathbf{W}$. Note that multiplication by a unitary matrix does not change the distribution of white Gaussain noise, that is, $\mathbf{W}$ and $\overline{\mathbf{W}}$ are identically distributed.

The SVD processing shown by Fig. 3 enables an MIMO OFDM system to adapt a suitable power/bit allocation to each subcarrier under constraints in a similar fashion of single input single output OFDM. However, in outdoor mobile environments, the MIMO channels can be quickly varied at each instant due to the mobility of mobile station. Therefore the SVD processing for the channel decomposition induces the burden complexity of the MIMO OFDM. But SFBC OFDM system turns the MIMO channel associated with each subcarrier into decoupled SISO channels without high complexity.

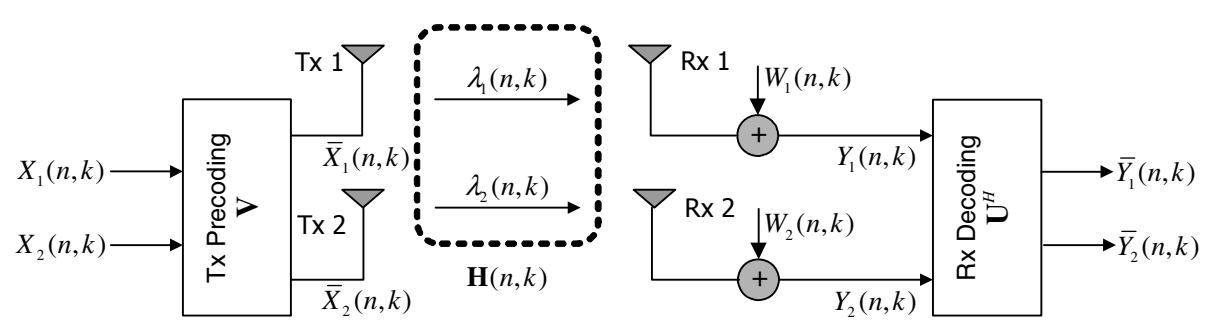

Fig. 3. An equivalent model of MIMO OFDM using SVD

\subsection{Parallel Decomposition in SFBC OFDM}

Equation (8) can be represented by the even and odd component vectors as follows

$$
\begin{aligned}
& \mathbf{Y}_{j, e}(n)=\mathbf{H}_{1 j, e}(n) \mathbf{X}_{1, e}(n)+\mathbf{H}_{2 j, e}(n) \mathbf{X}_{2, e}(n)+\mathbf{W}_{j, e}(n), \\
& \mathbf{Y}_{j, o}(n)=\mathbf{H}_{1 j, o}(n) \mathbf{X}_{1, o}(n)+\mathbf{H}_{2 j, o}(n) \mathbf{X}_{2, o}(n)+\mathbf{W}_{j, o}(n), \quad j=1,2 .
\end{aligned}
$$

This paper makes use of the following combining scheme at the receiver

$$
\begin{aligned}
& \tilde{\mathbf{X}}_{e}(n)=\mathbf{H}_{11, e}^{*}(n) \mathbf{Y}_{1, e}(n)+\mathbf{H}_{21, o}(n) \mathbf{Y}_{1, o}^{*}(n)+\mathbf{H}_{12, e}^{*}(n) \mathbf{Y}_{2, e}(n)+\mathbf{H}_{22, o}(n) \mathbf{Y}_{2, o}^{*}(n) \\
& \tilde{\mathbf{X}}_{o}(n)=\mathbf{H}_{21, e}^{*}(n) \mathbf{Y}_{1, e}(n)-\mathbf{H}_{11, o}(n) \mathbf{Y}_{1, o}^{*}(n)+\mathbf{H}_{22, e}^{*}(n) \mathbf{Y}_{2, e}(n)-\mathbf{H}_{12, o}(n) \mathbf{Y}_{2, o}^{*}(n) .
\end{aligned}
$$


Assuming the frequency responses between adjacent subcarriers are approximately constant, i.e.,

$$
\begin{aligned}
& \mathbf{H}_{11, e}(n) \approx \mathbf{H}_{11, o}(n) \\
& \mathbf{H}_{12, e}(n) \approx \mathbf{H}_{12, o}(n) \\
& \mathbf{H}_{21, e}(n) \approx \mathbf{H}_{21, o}(n) \\
& \mathbf{H}_{22, e}(n) \approx \mathbf{H}_{22, o}(n)
\end{aligned}
$$

Finally, the combined signals can be rewritten by

$$
\begin{aligned}
& \tilde{\mathbf{X}}_{e}(n)=\left(\left|\mathbf{H}_{11, e}(n)\right|^{2}+\left|\mathbf{H}_{12, e}(n)\right|^{2}+\left|\mathbf{H}_{21, e}(n)\right|^{2}+\left|\mathbf{H}_{22, e}(n)\right|^{2}\right) \mathbf{X}_{e}(n)+\tilde{\mathbf{W}}_{e}(n) \\
& \tilde{\mathbf{X}}_{o}(n)=\left(\left|\mathbf{H}_{11, o}(n)\right|^{2}+\left|\mathbf{H}_{12, o}(n)\right|^{2}+\left|\mathbf{H}_{21, o}(n)\right|^{2}+\left|\mathbf{H}_{22, o}(n)\right|^{2}\right) \mathbf{X}_{o}(n)+\tilde{\mathbf{W}}_{o}(n)
\end{aligned}
$$

where

$$
\begin{aligned}
& \tilde{\mathbf{W}}_{e}(n)=\mathbf{H}_{11, e}^{*}(n) \mathbf{W}_{1, e}(n)+\mathbf{H}_{21, o}(n) \mathbf{W}_{1, o}^{*}(n)+\mathbf{H}_{12, e}^{*}(n) \mathbf{W}_{2, e}(n)+\mathbf{H}_{22, o}(n) \mathbf{W}_{2, o}^{*}(n) \\
& \tilde{\mathbf{W}}_{o}(n)=\mathbf{H}_{21, e}^{*}(n) \mathbf{W}_{1, e}(n)-\mathbf{H}_{11, o}(n) \mathbf{W}_{1, o}^{*}(n)+\mathbf{H}_{22, e}^{*}(n) \mathbf{W}_{2, e}(n)-\mathbf{H}_{12, o}(n) \mathbf{W}_{2, o}^{*}(n) .
\end{aligned}
$$

The equation (15) which similar to equation (11) indicates that SFBC OFDM scheme turns the MIMO channel into decoupled SISO channels. This paper makes use of this equation for the adaptive modulation in MIMO OFDM.

\subsection{Adaptive Bit Loading}

In this paper, we consider the bit allocation scheme in [6]. We presuppose total transmit bits and load these bits onto each subcarrier in such a way that minimum energy is allocated to the entire transmission. Assume M-QAM is employed for each subcarrier, $b(n, k)$ bits per symbol are sent for the $k$-th subcarrier in the $n$-th OFDM symbol. According to [7][8], given the channel frequency response $H(n, k)$, the instantaneous bit error rate(BER) can be approximated by

$$
P_{e}(n, k)=c_{1} \exp \left\{-\frac{c_{2} \frac{E_{s}}{N_{0}}|H(n, k)|^{2}}{2^{b(n, k)}-1}\right\}
$$

where $c_{1}=0.2, c_{2}=1.6, E_{s}$ is the symbol energy at the transmitter, $N_{0}$ is the variance of $\mathbf{W}(n)$. This paper chooses the allocation scheme to achieve the target BER $\left(P_{r} \leq 10^{-2}\right)$ as follows 


$$
b(n, k)=\log _{2}\left[\frac{c_{2} \frac{E_{s}}{N_{0}}|H(n, k)|^{2}}{\ln \frac{c_{1}}{P_{r}}}+1\right]
$$

Let us consider the bit allocation in SFBC OFDM system. From the equation (15), the decoupled CSI for bit allocation is as follows

$$
|\mathbf{H}(n, k)|^{2}=\left|H_{11}(n, k)\right|^{2}+\left|H_{12}(n, k)\right|^{2}+\left|H_{21}(n, k)\right|^{2}+\left|H_{22}(n, k)\right|^{2}
$$

By substituting $|\mathbf{H}(n, k)|^{2}$ into $|H(n, k)|^{2}$ in equation (18), the bit allocation for SFBC OFDM is performed and the next procedures for complete bit allocation are based on Chow's method. Note that the allocated number of bits between subcarriers is exactly identical, i.e., $b(n, k)=b(n, k+1)$, because the frequency responses between adjacent subcarriers are approximately constant.

\section{Simulation Results}

\subsection{Simulation Parameters}

The parameters of adaptive OFDM system are as follows. Carrier frequency is $2 \mathrm{GHz}$ and the channels bandwidth is $20 \mathrm{MHz}$ which is divided equally among 2048 tones. The channel is based on COST 207 for a hilly terrain area [9] and the SISO channels associated with different couples of transmit/receive antennas are statistically equivalent and independent. The RMS delay spread is $5 \mu \mathrm{s}$. A guard interval of $22.6 \mu \mathrm{s}$ appended to each frame. A total of 4096 information bits transmitted in each OFDM frame, for an average 4 bits per subcarrier. Power allocation is uniformly performed. We assume that the total power from the two antennas for SFBC OFDM scheme is the same as the transmit power from the single transmit antenna. The velocities of mobile station are $60 \mathrm{~km} / \mathrm{h}$ and $100 \mathrm{~km} / \mathrm{h}$, respectively. We allocate $0,2,4$, or 6 bits to each subcarrier. So, each subcarrier is modulated using 4-, 16-, or 64-QAM, depending on the number of bits allocated. To compare with adaptive OFDM, the conventional OFDM scheme, called as an uniform OFDM, is uniformly modulated by 16-QAM. For the simulation according to feedback delay $T_{\Delta}$, the minimum feedback delay is $81 \mu \mathrm{s}$ and the maximum delay is $810 \mu \mathrm{s}$. For the purpose of the evaluation of various antenna schemes, three kinds of transmit(Tx)/ receive(Rx) antenna schemes are considered, i.e, 1Tx-1Rx, 2Tx-1Rx and 2Tx-2Rx, respectively.

\subsection{Performance in Case of Perfect CSI}

The perfect CSI for bit allocation can be referred as there is no feedback delay. It means very slow fading environment. Accordingly, the performance results show the evaluation of the efficiency of adaptive modulation scheme. Firstly, Fig. 4 shows the 
performance of uniform OFDM according to various $\mathrm{Tx} / \mathrm{Rx}$ antenna schemes. In comparison with $1 \mathrm{Tx}-1 \mathrm{Rx}$ antenna scheme, the power gain for $2 \mathrm{Tx}-1 \mathrm{Rx}$ antenna schemes at a BER of $10^{-3}$ is about $8 \mathrm{~dB}$. In case of $2 \mathrm{Tx}-2 \mathrm{Rx}$ scheme, the power gain at a BER of $10^{-3}$ is about $15 \mathrm{~dB}$. These results explain that a considerable diversity gain can be obtained when uniform OFDM system employs a diversity scheme. Now, from Fig. 5, we can observe the power gain of adaptive OFDM system employing a diversity scheme. The power gain for $2 \mathrm{Tx}-1 \mathrm{Rx}$ antenna scheme at a BER of $10^{-3}$ is about $1.5 \mathrm{~dB}$. In case of $2 \mathrm{Tx}-2 \mathrm{Rx}$ scheme, the power gain is about $5 \mathrm{~dB}$. From this result, the diversity gains in adaptive modulation are much less than those of uniform modulation. That is the reason why the adaptive modulation does very well deal with frequency-selectivity itself .

\subsection{Effect of the Outdated CSI in Mobile Situation}

Let us now shift the emphasis away from the perfect CSI to the outdated CSI. This raises the question of how the performance of adaptive OFDM system with diversity scheme appears in mobile fading environments. Fig. 6 indicates that the feedback delay has an effect on the performance of adaptive SFBC OFDM with $2 \mathrm{Tx} / 1 \mathrm{Rx}$ antenna scheme. As expected, the BER is gradually degraded as the feedback delay increases. This performance degradation is due to unavailability of CSI at the transmission time. In mobile situation, the CSI at the transmission instant is already the outdated information because the channel impulse response is timevarying. Let us examine that an adaptive OFDM system employing space-frequency block coding scheme is one of ways how to overcome the effects of feedback delay. As mentioned above, for the purpose of the evaluation the performance according to feedback delay, various simulations in terms of feedback delay are performed when the velocities are $60 \mathrm{~km} / \mathrm{h}$ and $100 \mathrm{~km} / \mathrm{h}$ respectively. To begin with, Fig. 7 indicates the simulation result when the feedback delay is $324 \mu$ s and $810 \mu$ s, respectively. Allowing for a BER of $10^{-3}$, let us compare the required power in Fig. 5 with that in Fig. 7. In case of $1 \mathrm{Tx}-1 \mathrm{Rx}$, let us compare with the perfect CSI of Fig. 5. An adaptive OFDM system with feedback delay needs the additional power more than about $8 \mathrm{~dB}$. It is shown that there is no merit of adaptive 1Tx-1Rx OFDM when feedback delay is long. On the other hand, in case of $2 \mathrm{Tx}-1 \mathrm{Rx}$ scheme, adaptive SFBC OFDM system with feedback delay needs the additional power of about $2 \mathrm{~dB}$ and $5 \mathrm{~dB}$, respectively. We can see that adaptive OFDM system employing a diversity scheme mitigates the effect of feedback delay. Also, adaptive SFBC OFDM system with $2 \mathrm{Tx}-2 \mathrm{Rx}$ scheme requires more than only $1 \mathrm{~dB}$. From this result, the performance degradation of adaptive OFDM due to the outdated CSI can be mitigated by diversity technique and we can refer the adaptive 2Tx-2Rx SFBC OFDM as an excellent system which have scarcely power loss in spite of severe feedback delay. Finally, let us see the performance according to the feedback delay. Fig. 8 indicates the performances of adaptive OFDM system along diversity scheme when the feedback delay is from $81 \mu$ s to the maximum $810 \mu$ s. SNR is $25 \mathrm{~dB}$ and the 
respective velocities are $60 \mathrm{~km} / \mathrm{h}$ and $100 \mathrm{~km} / \mathrm{h}$. Apparently we can see that the performance of adaptive OFDM with diversity schemes is much better than that of adaptive SISO OFDM.

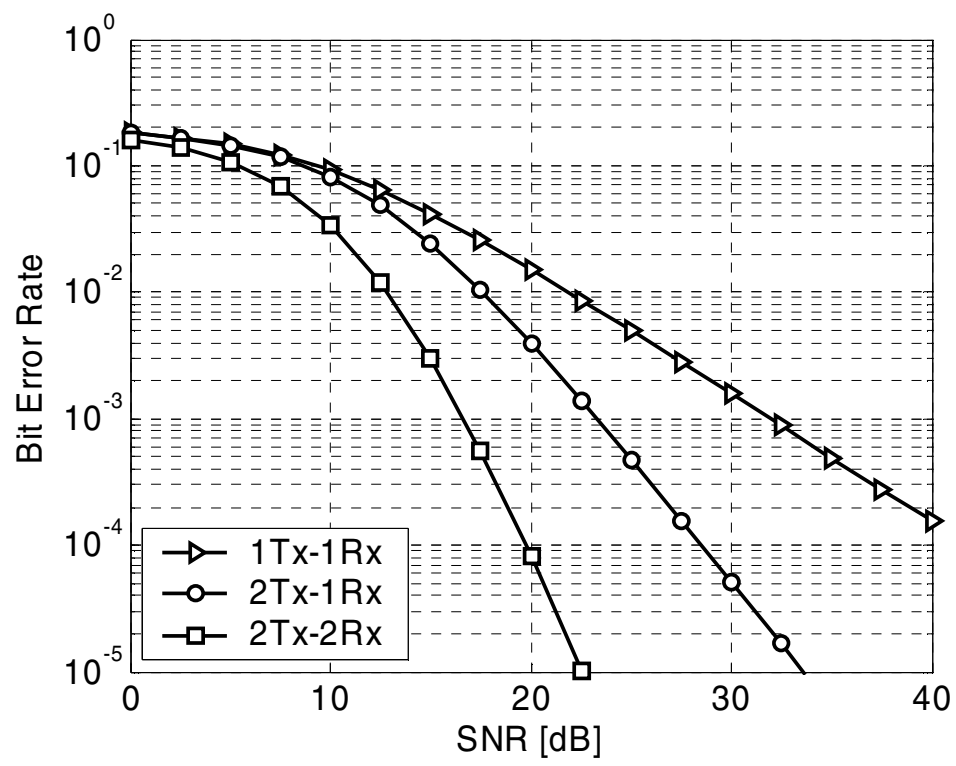

Fig. 4. BER curves of uniform OFDM according to Tx and Rx antenna schemes

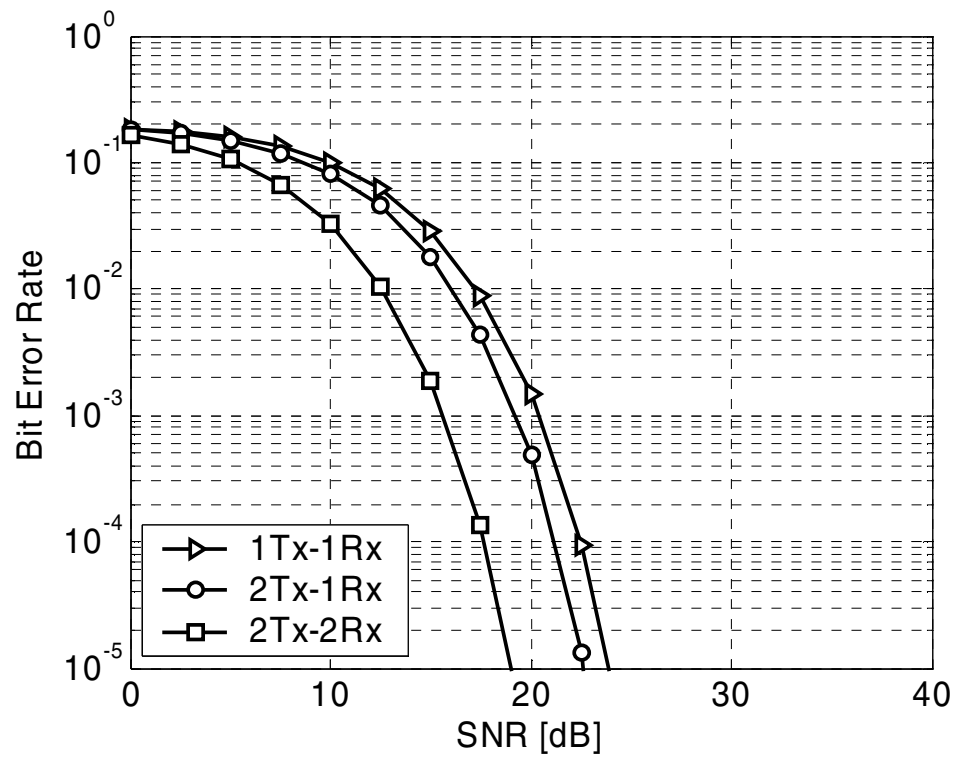

Fig. 5. BER curves of adaptive OFDM according to Tx and Rx antenna schemes 


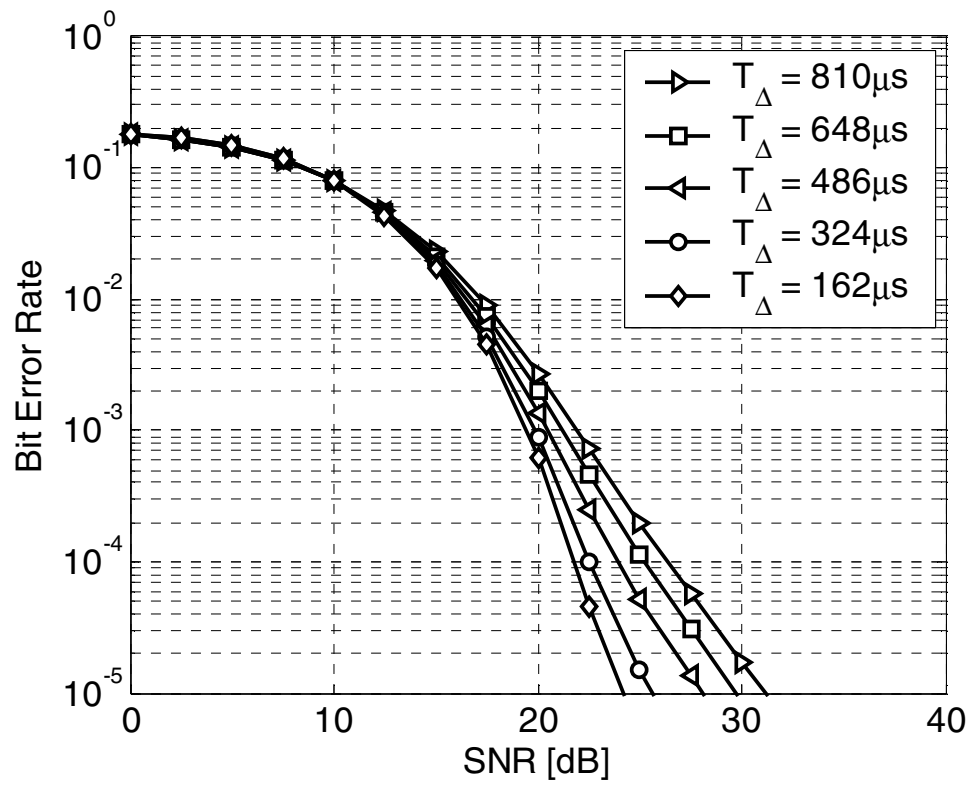

Fig. 6. BER curves according to feedback delay

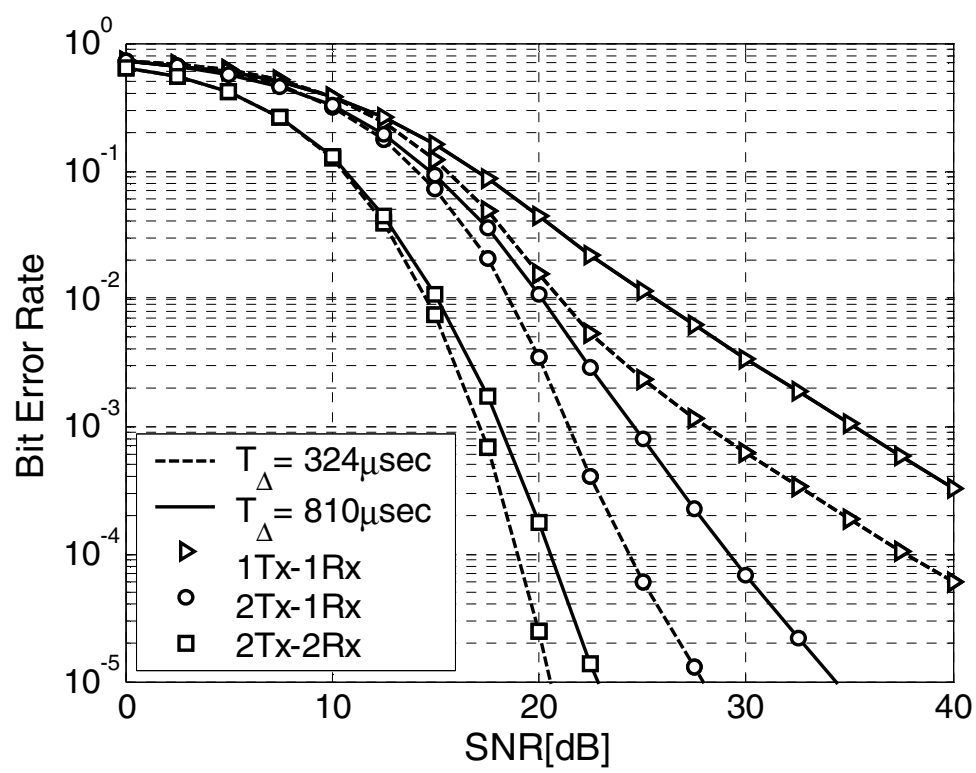

Fig. 7. BER curves according to diversity scheme in case that feedback delays are $324 \mu$ s and $810 \mu$ s respectively 


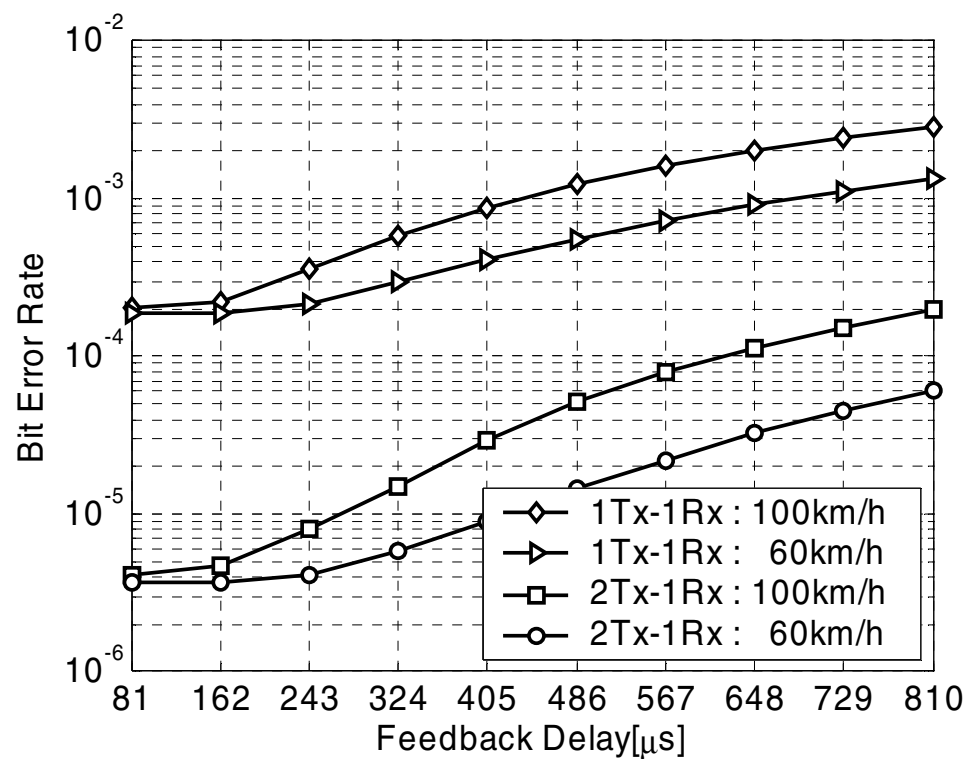

Fig. 8. BER curves according to feedback delay $\& \mathrm{Tx} / \mathrm{Rx}$ antenna schemes. $(25 \mathrm{~dB})$

\section{Conclusions}

In this paper combining an adaptive bit allocation scheme with SFBC OFDM system has been discussed. It has been illustrated that the CSI from SVD of MIMO channel is identical to the CSI from SFBC OFDM. From the various simulations, the performance of adaptive SFBC OFDM has been evaluated. Particularly, the BER performance according to the feedback delay has been indicated in detail. In the results, it is very interesting that the diversity schemes mitigate the effect of long feedback delay for adaptive OFDM. Most of all, adaptive SFBC OFDM with 2Tx$2 \mathrm{Rx}$ antenna scheme has made an excellent performance in spite of a severe feedback delay.

\section{References}

1. T. Keller and L. Hanzo, "Adaptive multicarrier modulation: a convenient framework for time-frequency processing in wireless communications," Proc. Of the IEEE, vol. 88, pp. 611-640, May 2000.

2. S. M. Alamouti, "A simple transmit diversity technique for wireless communications," IEEE J, Select. Areas Comm., vol. 16, pp. 1451-1458, Oct. 1998.

3. K. F. Lee and D. B. Williams, "A space-time coded transmitter diversity technique for frequency selective fading channels," in Proc. of the 2000 IEEE, SAMSP workshop, pp. 149-152.

4. H. Bolcskei and A. Paulraj, "Space-frequency coded broadband OFDM systems," in Proc. Of Wireless Comm. Networking Conf., pp. 1-6. Sept. 2000. 
5. Z. Liu, Y. Xin and G. B. Giannakis, "Space-time-frequency coded OFDM over frequencyselective fading channels," IEEE Trans. Sig. Proc., vol. 50, pp. 2465-2476, Oct. 2002.

6. P. S. Chow, J. M. Cioffi and J. A. C. Bingham, "A practical discrete multi-ton transceiver allocation algorithm for data transmission over spectrally shaped channels," IEEE Trans. on Comm, vol. 43, pp. 773-775, Apr 1995.

7. S. T. Chung and A. J. Goldsmith, "Degrees of freedom in adaptive modulation: a unified view," IEEE trans. On Comm., vol. 49, pp. 1561-1571, sep. 2001

8. S. Ye, R. S. Blum and L. L. Cimini, "Adaptive modulation for variable-rate OFDM systems with imperfect channel information," Proc, VTC 2002, pp. 767-771.

9. M. Patzold, Mobile Fading Channels, Wiley, 2002. 